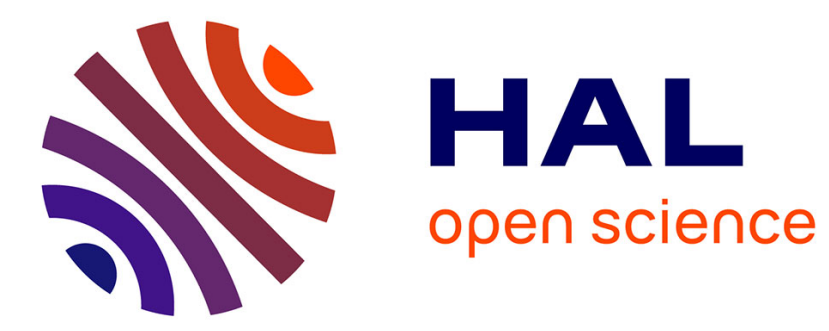

\title{
Living Lab MACVIA Handicap Correspondance
}

Isabelle Laffont, Claire Jourdan, Flavia Oana Coroian, Hubert Blain, V.

Carré, E. Viollet, I. Tavares, Charles Fattal, A. Gelis, F. Nouvel, et al.

\section{To cite this version:}

Isabelle Laffont, Claire Jourdan, Flavia Oana Coroian, Hubert Blain, V. Carré, et al.. Living Lab MACVIA Handicap Correspondance. La Presse Médicale, 2015, 44, pp.S60 - S69. 10.1016/j.lpm.2015.07.007 . hal-01755995

\section{HAL Id: hal-01755995 \\ https://hal.umontpellier.fr/hal-01755995}

Submitted on 6 Nov 2018

HAL is a multi-disciplinary open access archive for the deposit and dissemination of scientific research documents, whether they are published or not. The documents may come from teaching and research institutions in France or abroad, or from public or private research centers.
L'archive ouverte pluridisciplinaire HAL, est destinée au dépôt et à la diffusion de documents scientifiques de niveau recherche, publiés ou non, émanant des établissements d'enseignement et de recherche français ou étrangers, des laboratoires publics ou privés. 


\title{
Living Lab MACVIA Handicap
}

\author{
I. Laffont ${ }^{1,2}$, C. Jourdan ${ }^{1}$, F. Coroian ${ }^{1,2}$, H. Blain ${ }^{2,9}$, V. Carre ${ }^{1}$, E. Viollet ${ }^{3,4}$, I. Tavares ${ }^{1}$, C. Fattal ${ }^{20}$, A. Gelis ${ }^{7}$, \\ F. Nouvel ${ }^{4}$, K. Bakhti ${ }^{1,2}$, V. $\operatorname{Cros}^{1}$, K. Patte ${ }^{8}$, L. Schifano ${ }^{8}$, M. Porte ${ }^{3}$, E. Galano ${ }^{3}$, G. Dray ${ }^{11}$, M. Fouletier ${ }^{11^{1}}$, \\ F. Rivier ${ }^{12}$, R. Morales ${ }^{13}$, P. Labauge ${ }^{13}$, W. Camu ${ }^{13}$, B. Combe ${ }^{14}$, J. Morel $^{14}$, J. Froger ${ }^{2,3}$, B. Coulet ${ }^{15}$, \\ J. Cottalorda ${ }^{16}$, P. Kouyoumdjian ${ }^{17}$, 0. Jonquet ${ }^{18}$, L. Landreau ${ }^{18}$, H.-Y. Bonnin ${ }^{2,3}$, O. Hantkié ${ }^{19}$, P. Nicolas ${ }^{19}$, \\ M. Enjalbert ${ }^{10,20}$, C. Leblond $^{10}$, B. Soua ${ }^{21}$, P. Coignard ${ }^{20}$, D. Guiraud ${ }^{5,6}$, C. Azevedo $^{5,6}$, D. Mottet $^{2}$, P. Fraisse $^{5}$, \\ E. Pastor ${ }^{22}$, J. Mercier ${ }^{23}$, R. Bourret ${ }^{24}$, J. Bousquet ${ }^{25}$, J. Pélissier ${ }^{2,3}$, B. Bardy ${ }^{2}$, C. Herisson ${ }^{1,2}$, \\ A. Dupeyron ${ }^{2,3,4}$
}

1. CHRU de Montpellier, département de médecine physique et de réadaptation, 34090 Montpellier, France

2. Movement to Health (M2H), Euromov, université de Montpellier, Montpellier, France

3. CHU de Nîmes, hôpital Carémeau et du Grau du Roi, département de médecine physique et de réadaptation, 30029 Nîmes, France

4. CHU Carémeau, CEDMH, 30029 Nîmes, France

5. Université de Montpellier, laboratoire d'informatique, de robotique et de microélectronique de Montpellier, 34090 Montpellier, France

6. Institut national de recherche en informatique et en automatique, LIRMM, université de Montpellier, 34090 Montpellier, France

7. Centre Mutualiste Propara, 34000 Montpellier, France

8. Institut Marin Saint-Pierre, 34250 Palavas les Flots, France

9. CHRU de Montpellier, département de gériatrie, 34090 Montpellier, France

10. Centre Bouffard-Vercelli, 66290 Cerbère, France

11. École des Mines d'Alès, 30100 Alès, France

12. CHU de Montpellier, centre de référence Grand Sud des maladies neuromusculaires, département de neuropédiatrie, 34090 Montpellier, France

13. CHRU de Montpellier, département de neurologie, 34090 Montpellier, France

14. CHRU de Montpellier, département de rhumatologie, 34090 Montpellier, France

15. CHRU de Montpellier, département de chirurgie orthopédique, 34090 Montpellier, France

16. CHRU de Montpellier, département de chirurgie orthopédique et plastique infantile, 34090 Montpellier, France

17. CHU Carémeau, département de chirurgie orthopédique, 30029 Nîmes, France

18. CHRU de Montpellier, département de réanimation, 34090 Montpellier, France

19. Centre Bourgès, groupe Oc Santé, 34173 Castelneau-le-lez cedex, France

20. Association APPROCHE, CMRRF de Kerpape, BP 78, 56275 Ploemeur cedex, France

21. Association ADAGES, Les Fontaines d'ô, 34000 Montpellier, France

22. CCAS de Lattes, 34970 Lattes, France

23. CHRU de Montpellier, U1046 Inserm, université Montpellier 1, 34090 Montpellier, France

24. CHRU de Montpellier, Direction générale, 34090 Montpellier, France

25. MACVIA-LR, 34000 Montpellier, France

Correspondance :

Isabelle Laffont, CHU de Montpellier, hôpital Lapeyronie, département de médecine physique et de réadaptation, 191, boulevard du Doyen-Gaston-Giraud,

34295 Montpellier cedex 5, France.

i-laffont@chu-montpellier.fr 
Union européenne connaît un vieillissement démogra-

phique considérable. À compter de 2012, la population européenne en âge de travailler va commencer à se réduire, tandis que celle de plus de 60 ans poursuivra sa progression. Le partenariat européen d'innovation pour le vieillissement actif et en bonne santé (European Innovation Partnership on Active and Healthy Ageing [EIP on AHA], DG Sanco et DG CONECT) [1] vise à développer une approche globale axée sur une prise en charge optimisée pour identifier et éliminer les obstacles persistants à l'innovation pour un vieillissement actif et en bonne santé à travers des approches interdisciplinaires et intersectorielles. La région Languedoc Roussillon a été retenue comme site de référence de I'EIP on AHA (MACVIA-LR : contre les maladies chroniques pour un vieillissement actif et en bonne santé en Languedoc Roussillon) [2,3]. Les projets sous forme de Living Lab ont pour thème la prévention et la promotion du vieillissement actif et en bonne santé, les maladies chroniques [4-6], la prévention des chutes [7] et le handicap.

Cet article présente le Living Lab MACVIA Handicap (LLMH).

\section{Glossaire}

\section{AFM}

ALARME

APPROCHE

Association française contre les myopathies Association libre d'aide à la recherche sur la moelle épinière

\begin{tabular}{|c|c|}
\hline APPROCHE & $\begin{array}{l}\text { Association pour la promotion des nouvelles } \\
\text { technologies au service de personnes et situation } \\
\text { de handicap }\end{array}$ \\
\hline CCAS & Centre communal d'action sociale \\
\hline CEDMH & $\begin{array}{l}\text { Centre d'évaluation des dispositifs médicaux et du } \\
\text { handicap }\end{array}$ \\
\hline CENROB & $\begin{array}{l}\text { Centre d'expertise national en robotique (http:// } \\
\text { www.cenrob.org/) }\end{array}$ \\
\hline CCI & Chambre de commerce et d'industrie \\
\hline CNSA & Caisse nationale de solidarité pour l'autonomie \\
\hline EMA & École des Mines d'Alès \\
\hline EUROBIOMED & $\begin{array}{l}\text { Pôle de compétitivité filière santé, PACA- } \\
\text { Languedoc Roussillon }\end{array}$ \\
\hline EVAD & Évaluation du dispositif médical de Montpellier \\
\hline GIHP & $\begin{array}{l}\text { Groupement pour l'insertion des personnes } \\
\text { handicapées physiques }\end{array}$ \\
\hline HADE & Handicap, déficience motrice et performance \\
\hline INRS & $\begin{array}{l}\text { Institut national de recherche et de sécurité pour } \\
\text { la prévention des accidents du travail et des } \\
\text { maladies professionnelles }\end{array}$ \\
\hline I2ML & Institut méditerranéen des métiers de la longévit \\
\hline LIRMN & $\begin{array}{l}\text { Laboratoire d'informatique, de robotique et de } \\
\text { microélectronique de Montpellier }\end{array}$ \\
\hline LLMH & Living Lab MACVIA Handicap \\
\hline MPR & et de réadaptation \\
\hline MDPF & $\begin{array}{l}\text { Maisons départementales des personnes } \\
\text { handicapées }\end{array}$ \\
\hline SATT & Société d'accélération du transfert de technol \\
\hline Trans & $\begin{array}{l}\text { Agence régionale de l'innovation du Languedoc } \\
\text { Roussillon }\end{array}$ \\
\hline
\end{tabular}

\section{Le Living Lab MACVIA Handicap dans le contexte international, national et régional}

\section{Santé publique}

La population du Languedoc Roussillon est évaluée à 2,67 millions d'habitants (Insee 2011). Plus de $10 \%$ de la population régionale se trouve en situation de handicap, qu'il soit mental, moteur, psychique, auditif ou visuel [8]. Parmi ces personnes handicapées, la prévalence du handicap neurologique et locomoteur est mal connue, mais on sait qu'il figure parmi les toutes premières causes de handicap pour les personnes de moins de 60 ans dans notre pays (figure 1) [9]. On estime ainsi qu'il concerne plusieurs dizaines de milliers de personnes de moins de 60 ans en Languedoc Roussillon.

\section{Intégration dans l'EIP on AHA selon les plans d'action}

\section{Plan d'action $A 2$}

Le LLMH s'intègre dans le plan d'action sur la prévention des chutes (tableau I). Celles-ci concernent plus de $50 \%$ des personnes porteuses d'un handicap neurologique et/ou locomoteur. Leur fréquence dépend de l'étiologie et de la sévérité des déficiences [10]. Les actions du LLMH s'intègrent dans une stratégie pluridisciplinaire comprenant la prévention et le traitement des troubles neuro-orthopédiques et des pathologies dégénératives surajoutés, la préconisation et l'adaptation d'aides techniques au déplacement, la préconisation et l'adaptation d'aménagements de l'environnement personnel ou professionnel de la personne handicapée.

\section{Plan d'action A3}

Les actions du LLMH en matière de prévention de la malnutrition sont multiples avec, entre autres, la prévention de la dénutrition en lien avec les troubles de déglutition d'origine neurologique ou l'isolement social, la prévention de la surcharge pondérale et de l'obésité pour les personnes handicapées ayant un trouble de la marche ou pour celles se déplaçant en fauteuil.

\section{Plan d'action B3}

Le LLMH offre plusieurs exemples de prise en charge intégrée des soins pour les personnes handicapées. Ces prises en charge se structurent à partir de plusieurs établissements de santé qui offrent des bilans ambulatoires en hospitalisation de jour dédiés à ces patients. Ces bilans associent des prestations médicales et paramédicales multidisciplinaires, coordonnées par le médecin de médecine physique et de réadaptation (MPR), des prestations techniques dans le champ de l'aide technique en lien avec des industriels du secteur (exemple des cliniques du positionnement pour les patients en fauteuil), et des prestations médico-sociales dans lesquelles le rôle des associations d'usagers est déterminant. 


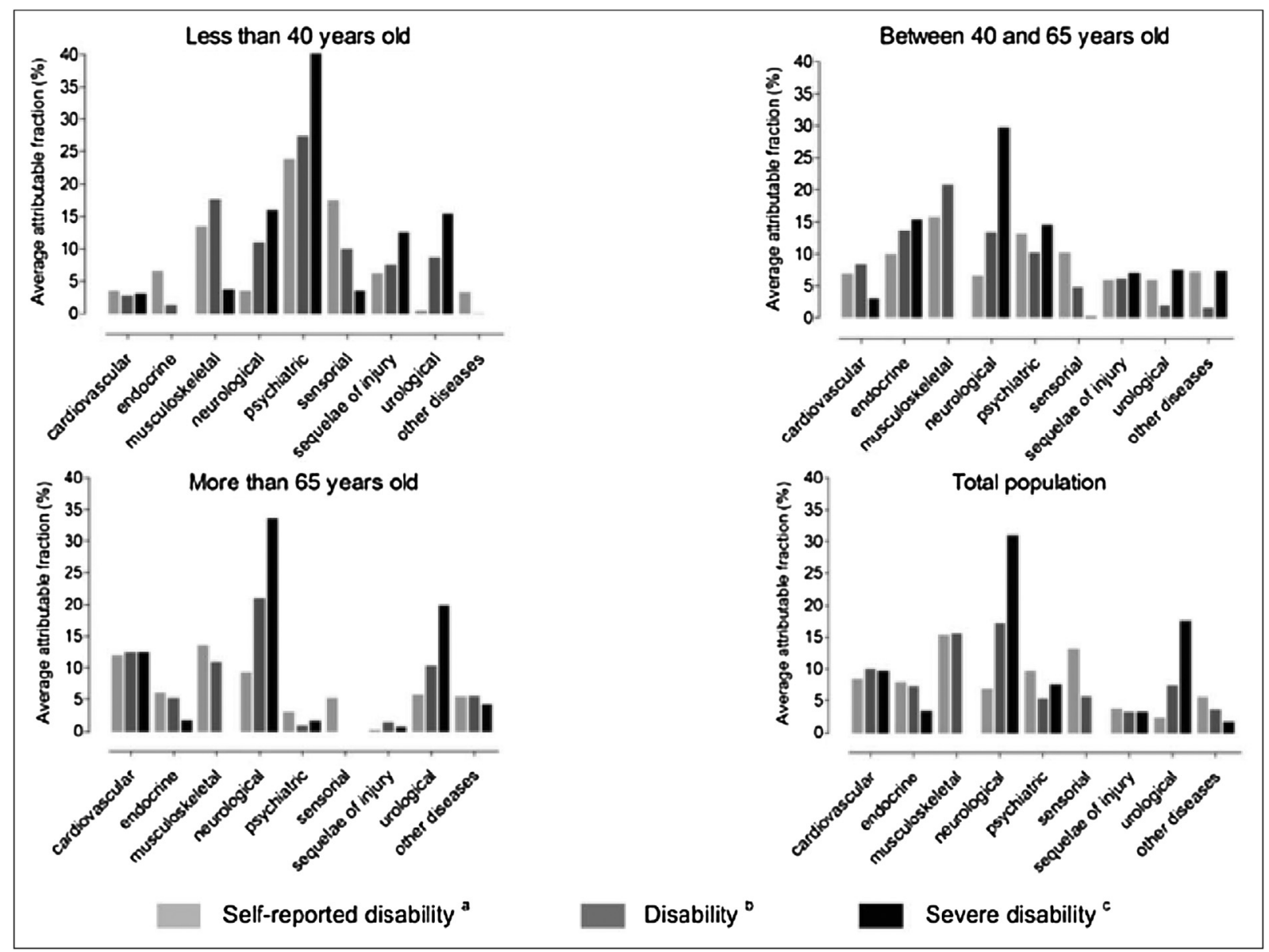

FIgURE 1

Imputabilité des principales classes de maladies sur le handicap en France, en fonction de l'âge des populations, du niveau d'incapacité ressentie, et du niveau de dépendance [9]

\section{Plan d'action C2}

La logique de vie autonome est centrale dans le LLMH. Elle repose sur les acteurs régionaux : établissements de santé, maisons départementales des personnes handicapées (MDPH), structures médico-sociales, services de soins infirmiers à domicile (SSIAD), service d'accompagnement à la vie sociale (SAVS), professionnels de santé libéraux, conseils généraux, Association de gestion du fond pour l'insertion professionnelle des personnes handicapées (AGEFIPH), Fonds pour l'insertion des personnes handicapées dans la fonction publique (FIPHFP). Plusieurs initiatives régionales en matière de nouvelles technologies illustrent la volonté de notre région de développer les outils technologiques dédiés au maintien à domicile et d'aide à la vie autonome [11].

\section{Plan d'action D4}

Le LLMH fédère de nombreuses initiatives locales en matière d'aménagements du logement et d'accessibilité permettant un maintien à domicile des personnes handicapées.

\section{Problématique régionale}

Notre région offre un cadre exceptionnel à la structuration du Living Lab MACVIA Handicap (figure 2).

Les établissements sanitaires publics ou privés de la région proposent une offre de soins reconnue dans la prise en charge aiguë des maladies neurologiques ou ostéo-articulaires.

La très forte implantation régionale de services de médecine physique et de réadaptation à orientation neurologique ou ostéo-articulaire favorise un dynamisme important dans la prise en charge des personnes handicapées : le LR dispose de 2,1 lits et places de soins de suite et de réadaptation pour 1000 habitants, ce qui le place au-dessus de la moyenne nationale à 1,7/1000 habitants. Par ailleurs, le maillage régional en matière de structures médico-sociales accueillant des personnes handicapées de moins de 60 ans est particulièrement dense, avec 4,8 places pour 1000 habitants alors qu'il n'est que de 4,1 au niveau national [12]. 
TABLEAU I

Intégration du LLMH dans les plans d'action de MACVIA

\begin{tabular}{|c|c|c|c|c|c|}
\hline & Plan d'action & Établissements de soin & Organismes publics & Organismes privés & $\begin{array}{l}\text { Structures universitaires } \\
\text { et établissements de formation }\end{array}$ \\
\hline $\mathrm{A} 2$ & Prévention des chutes & \multirow{4}{*}{$\begin{array}{c}\text { CHRU de Montpellier } \\
\text { CHU de Nîmes } \\
\text { Centre médical du Grau du Roi } \\
\text { Institut Marin Saint-Pierre } \\
\text { Centre Propara } \\
\text { Centre B.-Vercelli } \\
\text { Centre Bourgès } \\
\text { Hôpital Coste Floret } \\
\text { Centre Ster }\end{array}$} & $\begin{array}{l}\text { L'Etape } \\
\text { EVADIMM } \\
\text { CEDEMH }\end{array}$ & Digi-Health & \multirow{5}{*}{$\begin{array}{c}\text { Université de Montpellier } \\
\text { Pôle Rabelais } \\
\text { UFR Médecine } \\
\text { Euromov } \\
\text { LIRMM, INRIA } \\
\text { UFR STAPS } \\
\text { IUT de Nîmes } \\
\text { École des Mines d'Alès } \\
\text { IFMK } \\
\text { IFE }\end{array}$} \\
\hline A3 & Fragilité et malnutrition & & $\begin{array}{l}\text { Conseil général } \\
\text { Conseil régional }\end{array}$ & & \\
\hline B3 & $\begin{array}{c}\text { Prise en charge intégrée } \\
\text { et comorbidités }\end{array}$ & & & Digi-Health & \\
\hline C2 & Vie autonome & & $\begin{array}{c}\text { L'Etape } \\
\text { Eurobiomed } \\
\text { EVADIMM } \\
\text { CEDMH } \\
\text { Conseil général } \\
\text { Conseil régional } \\
\text { AGEFIPH, FIPHFP } \\
\text { Nîmes agglomération } \\
\text { Montpellier agglomération } \\
\text { CCI de Nîmes } \\
\text { CNSA, MDPH 30/34 } \\
\text { INRS } \\
\text { DIRECCTE }\end{array}$ & $\begin{array}{c}\text { SATT axLR } \\
\text { Transfert LR } \\
\text { Digi-Health }\end{array}$ & \\
\hline D4 & Environnement adapté & & & $\begin{array}{c}\text { SATT axLR } \\
\text { Transfert LR } \\
\text { Digi-Health }\end{array}$ & \\
\hline
\end{tabular}

FIPHFP : Fonds pour l'insertion des personnes handicapées dans la fonction publique ; AGEFIPH : Association de gestion du fond pour l'insertion professionnelle des personnes handicapées ; CEDMH : Centre d'évaluation des dispositifs médicaux et du handicap ; CNSA : Caisse nationale de solidarité pour l'autonomie ; DIRECCTE : Direction des entreprises, de la concurrence, de la consommation, du travail et de l'emploi ; EVADIMM : Évaluation du dispositif médical de Montpellier ; IFMK : Institut de formation en massokinésithérapie ; INRS : Institut national de recherche et de sécurité pour la prévention des accidents du travail et des maladies professionnelles ; IFE : Institut de formation en ergothérapie ; INRIA : Institut national de recherche en informatique appliquée ; LIRMM : Laboratoire d'informatique, de robotique et de microélectronique de Montpellier ; SATT : Société d'accélération du transfert de technologies; Transfert LR : Agence régionale de l'innovation du Languedoc Roussillon.

La prise en charge globale de la personne handicapée de moins de 60 ans revêt une importance toute particulière en Languedoc Roussillon.

\section{Objectifs}

Le LLMH regroupe des initiatives originales destinées à améliorer la prise en charge des personnes handicapées en intégrant des usagers, des acteurs du champ sanitaire, des acteurs du champ médico-social, et des industriels, en lien avec les organismes académiques d'enseignement et de recherche implantés dans la région.

L'idée est d'abord de mettre en place des organisations simplifiées permettant le suivi ambulatoire des personnes en situation de handicap et regroupant sur le même site un suivi médical et paramédical, un suivi médico-social, et un accès à des prestations très spécifiques autour des aides techniques. Ce suivi ambulatoire se structure souvent sous la forme de journées de bilan en hospitalisation de jour, sur les CHU de
Montpellier ou de Nîmes, voire dans certaines structures non universitaires.

Parallèlement, une dynamique très forte de recherche dans le champ des grandes déficiences sensori-motrices et des technologies appliquées au handicap existe en LR (technologies de I'information et de la communication et technologies du numérique, aides techniques de haute technologie, robotique de rééducation ou robotique d'assistance. . .). Plusieurs laboratoires de recherche développent des projets en lien avec cette thématique, voire se structurent intégralement autour d'elle comme le Centre européen de recherche Euromov [http:// www.euromov.eu/]. L'importance toute particulière que revêtent les technologies de rééducation ou de compensation pour ces patients stimule la recherche translationnelle, favorise le transfert technologique et les liens avec le tissu industriel régional, en appui sur des sociétés spécialisées comme la Société d'accélération du transfert de technologies, SATT AxLR, ou l'Agence régionale de l'innovation du Languedoc Roussillon (Transfert LR). 


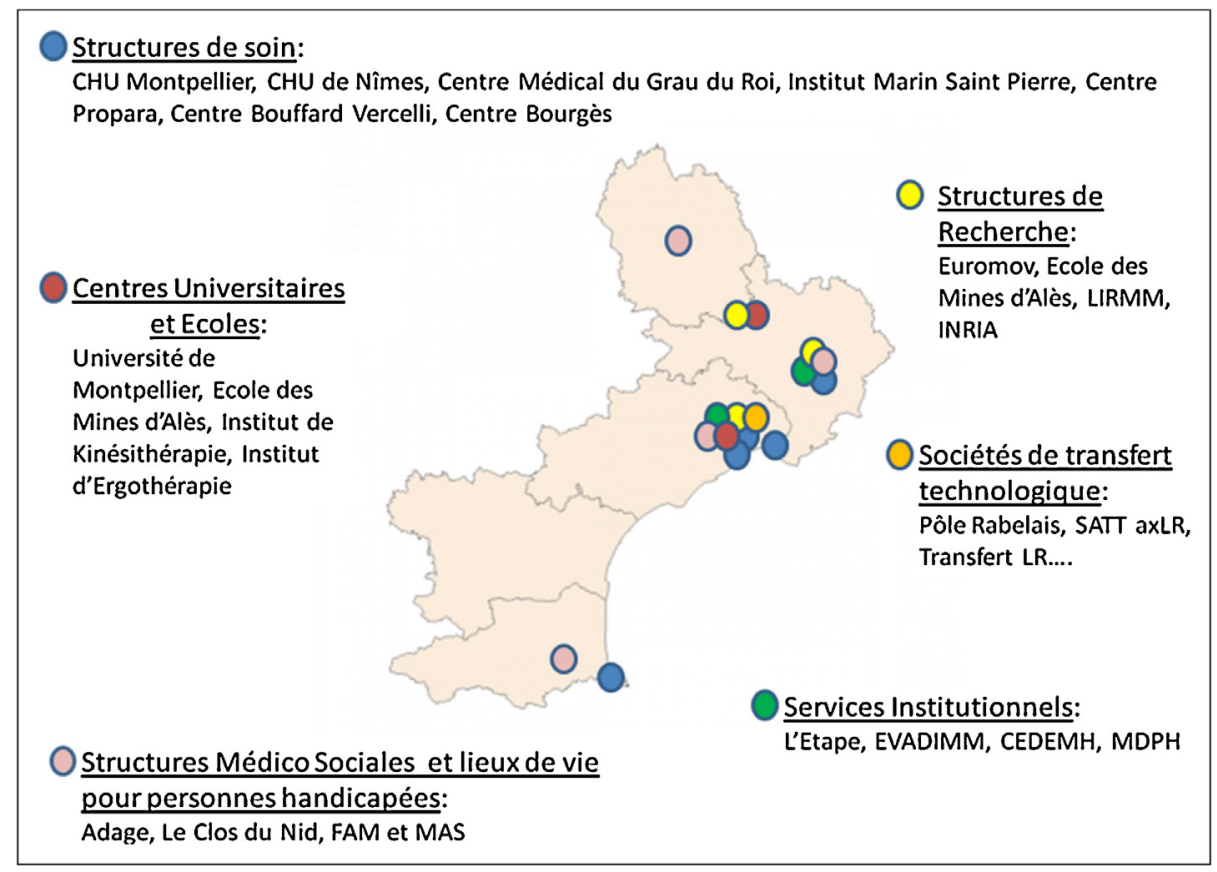

FIGURE 2

Localisation géographique des principales structures et groupes de structures impliquées dans le LL MACVIA Handicap

\section{Le Living Lab MACVIA Handicap}

\section{Activités de soins}

Les activités de soins du Living Lab MH reposent avant tout sur des bilans multidisciplinaires ambulatoires du handicap neurologique et locomoteur. Ces activités de bilan sont présentes dans plusieurs établissements sanitaires de la région, comme par exemple le secteur d'hospitalisation de jour du département de MPR du CHU de Montpellier, le centre d'évaluation HADEMOP du département de MPR du CHU de Nîmes, le secteur d'hospitalisation de jour du Centre Propara. Ces structures référentes entretiennent des liens étroits avec les établissements sanitaires et médico-sociaux de la région, mais aussi avec les réseaux de ville qui adressent très volontiers leurs patients pour un bilan complet (Union régionale des professionnels de santé, Montpellier). Elles associent régulièrement les associations d'usagers à la démarche de bilan proposée à ces patients (tableau II).

Elles offrent parfois des prestations très spécifiques, comme par exemple les « Cliniques du Positionnement au fauteuil roulant» (CHU de Nîmes, Centre Propara et Institut Marin Saint-Pierre), ou les centres de bilan en vue de la reprise de la conduite automobile (Centre du Grau du Roi, Centre Bouffard-Vercelli, Centre Propara). Enfin, certaines sont dédiées à des pathologies spécifiques comme les maladies neuromusculaires (Centre de référence Grand Sud intégrant le CHRU de Montpellier et le CHU de Nîmes, en lien très fort avec l'Association française des myopathies, AFM), la sclérose latérale amyotrophique (CHRU de Montpellier)...
Ces structures de bilans multidisciplinaires ambulatoires du handicap neurologique et locomoteur sont presque toujours adossées à des équipes de recherche avec lesquelles elles conduisent des projets de recherche sur les déficiences sévères de l'appareil neuro/locomoteur et sur le handicap. Elles sont en relation étroite et souvent contractualisée avec des industriels développant des dispositifs médicaux de rééducation ou de réadaptation (EVADIMM au CHU de Montpellier, CEDMH au CHU de Nîmes, CENROB au Centre Propara).

\section{Activités sociales}

Un très grand nombre d'associations à vocation médico-sociale existe dans la région [13]. La déclinaison régionale de la politique nationale de la Caisse nationale de solidarité pour l'autonomie (CNSA) se fait via les MDPH qui sont en liens très étroits avec les établissements sanitaires et médico-sociaux. Le LLMH a pour objectif de renforcer les échanges entre cette kyrielle de correspondants, qu'il s'agisse de structures étatiques ou de structures associatives, afin de simplifier le parcours de vie des personnes handicapées. Le Living Lab du Centre communal d'action sociale (CCAS) de Lattes (L'Etape) est très utile pour présenter les aides techniques aux usagers, en situation écologique.

\section{Associations de patients}

De nombreuses associations d'usagers sont impliquées dans les actions du LLMH. Elles constituent un relais d'information important pour les patients/usagers. Elles contribuent à un certain 
TAbleAu II

Principaux acteurs du Living Lab MACVIA Handicap

\begin{tabular}{|c|c|c|}
\hline Activité & Organisation & Description de l'activité \\
\hline \multirow[t]{6}{*}{ Soins } & CHRU de Montpellier & $\begin{array}{l}\text { Rééducation (MPR) des affections du système nerveux et de } \\
\text { l'appareil locomoteur. Bilans fonctionnels complexes ambulatoires du } \\
\text { handicap d'origine neurologique et ostéo-articulaire. Évaluation des } \\
\text { dispositifs médicaux (EVADIMM). Centre de référence maladies } \\
\text { neuromusculaires et sclérose latérale amyotrophique }\end{array}$ \\
\hline & CHU de Nîmes & $\begin{array}{c}\text { Rééducation (MPR) des affections du système nerveux, de l'appareil } \\
\text { locomoteur, rééducation cardiologique, rééducation vésico- } \\
\text { sphinctérienne. Bilans fonctionnels complexes ambulatoires du } \\
\text { handicap d'origine neurologique et ostéo-articulaire (HADEMOP). } \\
\text { Évaluation des dispositifs médicaux (CEDMH). Clinique du } \\
\text { positionnement pour patients en fauteuil roulant. Bilans conduite } \\
\text { automobile personnes handicapées }\end{array}$ \\
\hline & Institut Marin Saint-Pierre & $\begin{array}{c}\text { Rééducation (MPR) pédiatrique. Bilan du handicap moteur et cognitif } \\
\text { de l'enfant. Clinique du positionnement pour patients en fauteuil } \\
\text { roulant }\end{array}$ \\
\hline & Centre Propara & $\begin{array}{c}\text { Rééducation (MPR) des patients blessés médullaires. Bilans } \\
\text { fonctionnels complexes ambulatoires du handicap d'origine } \\
\text { neurologique. Centre d'expertise national en robotique (CENROB). } \\
\text { Clinique du positionnement pour patients en fauteuil roulant. Bilans } \\
\text { conduite automobile personnes handicapées }\end{array}$ \\
\hline & Centre B.-Vercelli & $\begin{array}{c}\text { Rééducation (MPR) des affections du système nerveux et de } \\
\text { l'appareil locomoteur. Bilans conduite automobile personnes } \\
\text { handicapées }\end{array}$ \\
\hline & Centre Bourgès (0c Santé) & Rééducation (MPR) des affections du système nerveux \\
\hline \multirow[t]{7}{*}{ Formation } & UFR médecine Montpellier-Nîmes & $\begin{array}{l}\text { Enseignement aux étudiants en médecine (module transversal } \\
\text { "Rééducation-Maladies Chroniques "). Enseignement troisième cycle } \\
\text { des études médicales (internes de MPR). Enseignement post- } \\
\text { universitaire en lien avec la thématique Handicap. Enseignement de } \\
\text { l'Orthophonie }\end{array}$ \\
\hline & UFR STAPS & Formation des moniteurs en activités physiques adaptées \\
\hline & Institut de formation en ergothérapie & $\begin{array}{l}\text { Formation des ergothérapeutes. Animation du congrès national des } \\
\text { ergothérapeutes (« Expériences en ergothérapie ») }\end{array}$ \\
\hline & Institut de formation en masso-kinésithérapie & Formation des masseurs kinésithérapeutes \\
\hline & École des Mines d'Alès & $\begin{array}{l}\text { Projets thématiques, soutien aux entreprises et incubateur } \\
\text { technologique dédié au Handicap }\end{array}$ \\
\hline & IUT de Nîmes & $\begin{array}{l}\text { Départements Génie Civil/Génie Mécanique. Accompagnement de } \\
\text { projets. Incubation de jeunes entreprises }\end{array}$ \\
\hline & $\begin{array}{l}\text { Ingénieurs TIC Santé } \\
\text { Congrès thématiques annuels }\end{array}$ & Congrès RARE, Congrès des EMPR, Digiworld Summit \\
\hline \multirow{7}{*}{$\begin{array}{l}\text { Liens avec les acteurs } \\
\text { institutionnels }\end{array}$} & Région LR & Porteur du Projet MacVia-LR Handicap \\
\hline & Agence régionale de santé $L R$ & $\begin{array}{c}\text { Soutien aux projets thématiques } \\
\text { Financement de projets dédiés à la thématique Handicap, } \\
\text { labellisation de projets d'Éducation à la Santé dans le champ du } \\
\text { Handicap }\end{array}$ \\
\hline & CCAS de Lattes : l'Etape & Démonstration, essais et préconisation d'aides techniques \\
\hline & CCI de Nîmes & Accompagnement de projets de développement technologique \\
\hline & $\begin{array}{l}\text { Municipalités en LR, Nîmes agglomération } \\
\text { et Montpellier agglomération }\end{array}$ & Organisations territoriales, soutien aux projets thématiques \\
\hline & MDPH Gard et Hérault, CNSA & $\begin{array}{c}\text { Accompagnement des patients et de leurs familles dans leurs projets } \\
\text { personnel, familial et professionnel }\end{array}$ \\
\hline & Cap Emploi, AGEFIPH, Association COMETE & $\begin{array}{l}\text { Accompagnement des patients dans leurs démarches de retour } \\
\text { à l'emploi ou de maintien dans l'emploi }\end{array}$ \\
\hline
\end{tabular}




\section{TABLEAU II (Suite).}

\begin{tabular}{|c|c|c|}
\hline Activité & Organisation & Description de l'activité \\
\hline \multirow[t]{8}{*}{ Recherche } & Centre Euromov/EA2991 & $\begin{array}{c}\text { Recherche translationnelle : déficiences, plasticité des systèmes } \\
\text { sensorimoteurs et rééducation, technologies appliquées à la } \\
\text { rééducation/réadaptation }\end{array}$ \\
\hline & LIRMM/INRIA & $\begin{array}{l}\text { Travaux en lien avec des cliniciens sur la robotique d'assistance et/ } \\
\text { ou la stimulation électrique fonctionnelle, travaux sur les } \\
\text { technologies numériques dédiées à la rééducation }\end{array}$ \\
\hline & École des Mines d'Alès & $\begin{array}{c}\text { Projets thématiques « Handicap » portés par des ingénieurs et } \\
\text { développés en lien avec les usagers, soutien aux jeunes entreprises, } \\
\text { incubateur technologique orienté vers les technologies appliquées au } \\
\text { handicap }\end{array}$ \\
\hline & Epsylon/EA4556 & Recherche prévention santé \\
\hline & Santé SIH/EA4614 & Recherche en santé et éducation, situations de handicap \\
\hline & CHRU de Montpellier et CHU de Nîmes & $\begin{array}{c}\text { Recherche clinique thématique, recherche sur les dispositifs } \\
\text { médicaux dédiés à la rééducation et/ou à la compensation/ } \\
\text { assistance }\end{array}$ \\
\hline & IFRH & $\begin{array}{c}\text { Implication forte des acteurs régionaux dans I'Institut fédératif de } \\
\text { recherche sur le handicap }\end{array}$ \\
\hline & Labex NUMEV & $\begin{array}{l}\text { Solutions numériques, matérielles et modélisation pour } \\
\text { l'environnement et le vivant : implication forte des acteurs régionaux } \\
\text { dans l'axe " Aide à la personne malade ou déficiente » }\end{array}$ \\
\hline Lien avec les associations d'usagers & $\begin{array}{l}\text { APF, AFM, AFTC, GLIP, France AVC, AFSEP, } \\
\text { France Parkinson, ALIS, ALARME, GIHP }\end{array}$ & $\begin{array}{l}\text { Implication dans des actions sanitaires, implication dans des projets } \\
\text { de recherche cliniques et technologiques, soutien à plusieurs projets } \\
\text { thématiques }\end{array}$ \\
\hline \multirow[t]{4}{*}{ 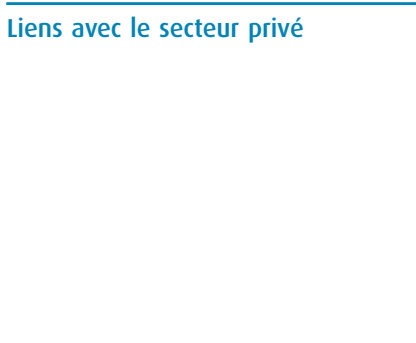 } & SATT AxLR & $\begin{array}{l}\text { Maturation et commercialisation de produits innovants issus de la } \\
\text { recherche académique }\end{array}$ \\
\hline & Transfert LR & Animation territoriale et accompagnement de projets innovants \\
\hline & Eurobiomed & $\begin{array}{l}\text { Cluster au service du développement des activités innovantes dans le } \\
\text { domaine de la santé : soutien aux projets thématiques, liens entre } \\
\text { les partenaires institutionnels, académiques et les entreprises }\end{array}$ \\
\hline & I2ML & $\begin{array}{c}\text { Fondation partenariale de l'université de Nîmes. Accompagnement } \\
\text { à l'émergence d'une nouvelle filière économique autour du maintien } \\
\text { à domicile }\end{array}$ \\
\hline \multirow[t]{2}{*}{$\begin{array}{l}\text { Liens avec les sociétés savantes et } \\
\text { les organismes de recherche }\end{array}$} & Sofmer & $\begin{array}{c}\text { Les équipes de médecine physique et de réadaptation du Languedoc } \\
\text { Roussillon sont très actives au sein de la Sofmer et s'impliquent dans } \\
\text { plusieurs actions collectives dans le cadre de la politique scientifique } \\
\text { de la Société savante }\end{array}$ \\
\hline & IFRH & $\begin{array}{l}\text { Deux laboratoires de recherche de la Nouvelle Université de } \\
\text { Montpellier sont membres de I'IFRH (EA } 4614 \text { et EA 2991), } \\
\text { respectivement dans l'axe « Recherches en santé publique et } \\
\text { sciences humaines et sociales » et dans l'axe " Technologies } \\
\text { appliquées aux grandes déficiences et au handicap » }\end{array}$ \\
\hline
\end{tabular}

AGEFIPH : Association de gestion du fond pour l'insertion professionnelle des personnes handicapées ; cCAS : Centre communal d'action sociale ; CNSA : Caisse nationale de solidarité pour l'autonomie; INRIA : Institut national de recherche en informatique appliquée ; LIRMM : Laboratoire d'informatique, de robotique et de microélectronique de Montpellier ; SATT : Société d'accélération du transfert de technologies; Transfert LR : Agence régionale de l'innovation du Languedoc Roussillon.

nombre d'actions d'éducation destinées aux personnes et à leur entourage, à travers des réunions thématiques ou des journées d'information $[14,15]$. Elles sont partenaires de certaines organisations sanitaires et contribuent parfois à leur financement. Enfin, elles peuvent être impliquées très en amont lors de la mise en place de projets de recherche, notamment dans le champ des aides techniques où les patients doivent être impliqués tout au long du processus de développement et de validation technologique. Parmi les associations d'usagers impliquées dans le LLMH, on peut citer de façon non exhaustive : APF, AFM, AFTC, GLIP, France AVC, AFSEP, France Parkinson, ALIS, ALARME, GIHP... 


\section{Enseignement}

La thématique du handicap d'origine neurologique et ostéo-articulaire est au cœur de nombreux enseignements à la faculté de médecine Montpellier-Nîmes : module spécifique d'enseignement dans le second cycle des études médicales, enseignements de troisième cycle destinés aux internes futurs spécialistes de MPR, diplômes universitaires. Cette thématique est également fortement représentée dans le cursus des filières paramédicales de rééducation : masso-kinésithérapie, ergothérapie, orthophonie, podo-orthèse, ortho-prothèse. . . Elle imprègne largement les enseignements prodigués dans la filière des ingénieurs TIC Santé ou des Masters Activités physique adaptées de la Nouvelle Université de Montpellier, dans lesquels interviennent de nombreuses équipes médicales, dont les équipes de MPR.

L'École des Mines d'Alès (EMA) est un exemple éloquent de développement d'un projet pédagogique et de recherche résolument tourné vers la personne handicapée. L'EMA a su mettre ses compétences au service des personnes handicapées à travers des projets concrets portés par des étudiants en liens étroits avec les usagers, mais aussi à travers un soutien aux entreprises et la création d'un incubateur technologique orientés vers les technologies appliquées au handicap.

Plusieurs congrès thématiques d'envergure nationale ou internationale se déroulent de façon régulière sur le site de Montpellier-Nîmes : congrès RARE (rencontres Eurobiomed autour des Maladies rares), congrès des EMPR qui a une vocation affichée de formation professionnelle, Digiword Summit (journée DIGIHEALTH), congrès National d'Ergothérapie...

\section{Recherche}

Le dynamisme autour de la thématique des grandes déficiences de l'appareil locomoteur et du handicap nourrit de nombreux projets de recherche de dimension nationale ou internationale. Le centre Euromov, dont le cœur est constitué par le laboratoire Movement to Health (M2H) de I'Université de Montpellier, récemment rejoint par l'École des Mines d'Alès, coordonne avec les équipes cliniques des CHU de Nîmes et de Montpellier de nombreux projets de recherche translationnelle en lien avec cette thématique : déficiences motrices dans les suites d'un accident vasculaire cérébral et plasticité des systèmes sensori-moteurs [16-20], plasticité des adaptations posturales dans la lombalgie chronique [21], troubles de la motricité et rééducation par le rythme dans la maladie de Parkinson [22].

La recherche sur les dispositifs médicaux, qui incluent les aides techniques de compensation des grandes déficiences et les technologies de rééducation, est également très active dans la région. Elle est structurée à travers des plateformes dédiées sur les deux CHU. Le CHU de Montpellier propose la plateforme EVADIMM qui offre aux équipes cliniques, aux industriels et aux chercheurs, un guichet unique d'information et d'accompagnement de projets de recherche sur les dispositifs médicaux. Plus de 150 projets de recherche sur les dispositifs médicaux sont ainsi recensés sur le CHU de Montpellier. Plusieurs reposent sur l'expertise des équipes de MPR dans l'évaluation des dispositifs médicaux dédiés à la compensation des grandes déficiences [23-25]. Cette plateforme entretient des liens forts avec son environnement régional : Transfert $L R$, pôle de compétitivité Eurobiomed, SATT AxLR, conseil régional... Le CHU de Nîmes a mis en place le CEDMH, structure de recherche dédiée à l'évaluation des dispositifs médicaux destinés aux personnes en situation de handicap. Son rôle est à la fois le co-développement et l'évaluation clinique de dispositifs médicaux non invasifs destinés à des patients en situation de handicap. Cette structure a permis d'accélérer le transfert de connaissances vers l'autonomie ou le confort de la personne handicapée. Ceci a permis de favoriser les dépôts de co-brevets $\mathrm{CHU}$-industrie et de finaliser des études de qualité comparant l'efficacité de dispositifs existants ou de prototypes reposant sur des critères d'évaluation robustes (indices de pression pour les matelas, étude cinématique pour les guidons de transfert). Ces travaux sont réalisés grâce aux liens forts entre l'École des Mines d'Alès et sa structure d'incubation (Innov'up), les sections génie civil et génie mécanique de l'IUT de Nîmes, la participation de l'Institut national de recherche et de sécurité pour la prévention des accidents du travail et des maladies professionnelles (INRS), du laboratoire M2H d'Euromov et le soutien de la CNSA. Il s'inscrit dans la dynamique locale de la communauté d'agglomération de Nîmes, de la CCl de Nîmes, du plan 2014-2020 de 3S en LR et des actions de la Directe.

D'autres laboratoires implantés dans la région ont des liens très forts avec les cliniciens sur la thématique des grandes déficiences motrices, liens qui s'expriment à travers plusieurs projets de recherche emblématiques sur le sujet : l'équipe DEMAR de I'Institut national de recherche en informatique appliquée (INRIA) sur le développement d'un outil de stimulation électrique fonctionnelle du blessé médullaire [26] ou de l'hémiplégique [27], le Laboratoire d'informatique, de robotique et de microélectronique de Montpellier (LIRMM) sur le développement des jeux vidéo de rééducation [28], I'École des Mines d'Alès qui travaille avec les équipes de MPR des CHU et le centre EUROMOV sur le développement des interfaces cérébrales dans le champ de la neuro-rééducation [29,30].

Le laboratoire Movement to Health (Euromov) et le laboratoire SanteSih (Santé et Éducation, Situation de Handicap) sont des membres actifs de l'Institut fédératif de recherche sur le handicap (http://ifr-handicap.inserm.fr/). Movement to Health participe à la gouvernance du Laboratoire d'Excellence NUMEV (http://www.lirmm.fr/numev/) en s'impliquant tout particulièrement dans l'axe "Santé : aide à la personne malade ou déficiente » du Labex.

\section{École des Mines d'Alès}

Créée en 1843, pour former les cadres de l'industrie minière, l'École des Mines d'Alès s'est complètement renouvelée dans 
les années $1980^{26}$ pour accompagner la mutation profonde des entreprises, répondre à leurs nouveaux besoins en créant un incubateur technologique pour mettre la créativité de ses élèves au service de la cause des personnes en situation de handicap. La recherche relève de trois thématiques: les matériaux (C2MA : Centre des matériaux des Mines d'Alès), I'environnement et les risques (LGEI : laboratoire de génie de l'environnement industriel) et les technologies de l'information et de la communication (LGI2P : Laboratoire de génie informatique et ingénierie de production).

Dès la création de son laboratoire de mécanique, l'EMA a été sollicitée par de jeunes actifs en situation de handicap, désireux de vivre en autonomie, et qui demandaient l'amélioration des équipements existants ou le développement de nouveaux produits : un architecte souhaitant un fauteuil de faible encombrement pour faciliter ses voyages en avion, un agriculteur qui avait besoin d'un fauteuil « tout terrain » pour continuer à exercer sa profession, un sportif de haut niveau désireux de continuer sa pratique du ski après son accident... Ces réalisations ont été suivies de beaucoup d'autres, plus ambitieuses encore, lorsque I'EMA a disposé d'une plateforme de mécatronique, dotée de moyens d'étude, de conception et de fabrication étendus : adaptation d'une prothèse tibiale pour le handisport, fauteuil tout terrain motorisé, adaptation des commandes d'un kart...

Avec la généralisation des technologies de l'information et de la communication, les dispositifs d'aide aux personnes en situation de handicap se diversifient et touchent de nouveaux domaines. C'est ainsi que le Laboratoire LGI2P met ses compétences en génie logiciel à contribution dans un projet collaboratif de domotique auto-adaptative pour favoriser un maintien à domicile de qualité ; projet est conduit en lien avec l'I2ML ${ }^{27}$ et le LIRMM $^{28}$, notamment... Le LGI2P collabore également à un programme de recherche consacré au suivi et à la rééducation post-AVC en partenariat avec le centre EUROMOV ${ }^{29}$ et l'Université de Maynooth, en Irlande. Enfin, le LGIZP participe au projet AUREVI, porté par I'Institut ARAMAV, faisant appel aux STIC pour lutter contre le handicap visuel.

L'incubateur technologique de l'EMA, créé en 1984, accueille des projets en lien avec les thématiques des laboratoires. L'incubateur a ainsi permis la création d'entreprises dans le domaine du handicap et de la santé, telles que: Le Griot ${ }^{30}$, Cairpol ${ }^{31}$,

26 http://www.annales.org/archives/theseAles.html : résumé de la thèse de doctorat en histoire de Cécile Turion, consacrée à l'histoire de l'École des mines d'Alès.

27 http://www.i2ml.fr : Institut méditerranéen des métiers de la longévité.

28 http://www.lirmm.fr : Laboratoire d'informatique, de robotique et de microélectro-

nique de Montpellier.

29 http://www.euromov.eu.

30 http://www.le-griot.com/ : matériel de facilitation de l'écoute en audiodescription à destination des malvoyants.

31 http://www.cairpol.com/index.php?lang=fr : systèmes miniatures de surveillance de la qualité de l'air, destinés à l'origine aux personnes fragiles.
Bodydens $^{32}$, Ostéorêve ${ }^{33} \ldots$ Parmi les projets en cours de développement, on peut citer, notamment, les projets S\&] Technologies (dispositif médical pour la détection et la prévention de chutes liées à la maladie de Parkinson) et UBIMOV (logiciel de détection de situations potentiellement à risques pour les résidents des EHPAD).

Les missions de l'EMA ont développé la pédagogie entrepreneuriale de formation-action avec l'appui d'HEC-Entrepreneur ${ }^{34}$. Les élèves ont réalisé différents services innovants destinés aux personnes en situation de handicap, comme par exemple un déambulateur permettant à des enfants déficients intellectuels de participer aux jeux de leur âge.

\section{Liens avec l'industrie}

Les liens avec le tissu industriel régional ou national sont forts et se structurent de façon accélérée depuis une dizaine d'années. Les organismes spécialisés dans le transfert technologique (SATT AxLR, Transfert $L R$ ), les incubateurs régionaux et le pôle de compétitivité Eurobiomed facilitent la mise en relation entre industriels et organismes publics, établissements de soin et établissements de formation.

Les liens avec l'industrie s'expriment à travers des partenariats de recherche qui vont des phases de Recherche \& Développement à l'évaluation clinique en situation de vie. L'exemple de la jeune société NaturalPad est une illustration exemplaire de cette structuration en Living Lab régionale, avec une implantation forte dans les établissements de soin, une incubation au sein d'Euromov et plusieurs projets de recherche en cours sur les jeux vidéo de rééducation.

\section{Interactions régionales, nationales et internationales du Living Lab}

Le concept de Living Lab Santé et Autonomie a été défini par un rapport du ministère de l'Industrie en 2011 [31]. Ces initiatives se structurent en Europe à travers des organisations, comme par exemple le réseau ENOLL (The European Network of Living Labs - http://www.openlivinglabs.eu/). Le Living Lab MH répond au cahier des charges proposé par ce réseau avec la particularité d'avoir localement une implication forte des équipes cliniques qui fait parfois défaut dans les Living Lab autonomie-santé. Par ailleurs, il regroupe sur une même région un très grand nombre de structures publiques et privées susceptibles de se structurer dans ce sens en mettant en commun leurs moyens et leurs savoir-faire.

Déclaration d'intérêts : les auteurs déclarent ne pas avoir de conflits d'intérêts en relation avec cet article.

\footnotetext{
32 http://www.bodysens.com/ : systèmes de monitoring sans fil, utilisés, par exemple, pour les prématurés.

33 http://www.osteoreve.com/ : matelas anatomique personnalisé.

34 http://www.hec.fr/Masteres-Specialises/Programmes/Entrepreneurs/Pedagogie.
} 


\section{Références}

[1] Bousquet J, Michel JP, Standberg T, Crooks G Iakovidis I, Iglesia M. The European Innovation Partnership on Active and Healthy Ageing: the European Geriatric Medicine introduces the EIP on AHA Column. Eur Geriatr Med 2014;5:361-2.

[2] Bousquet J, Hajjam J, Piette F, Jean-Bart B, Wlosik C, Robine JM, et al. [The French reference sites of the European Innovation Partnership on active and healthy ageing]. Presse Med 2013;42:1558-61.

[3] Bousquet J, Bourquin C, Augé P, Domy P, Bringer J, Camuzat T, et al. MACVIA-LR reference site of the European Innovation Partnership on Active and Healthy Ageing. Eur Geriatr Med 2014;5:406-15.

[4] Bousquet J, Jorgensen C, Dauzat M, Cesario A, Camuzat T, Bourret R, et al. System medicine approaches for the definition of complex phenotypes in chronic diseases and ageing. From concept to implementation and policies. Curr Pharm Des 2014;20:592844.

[5] Bousquet J, Tanasescu CC, Camuzat T, Anto IM, Blasi F, Neou A, et al. Impact of early diagnosis and control of chronic respiratory diseases on active and healthy ageing. A debate at the European Union Parliament. Allergy 2013;68:555-61.

[6] Bousquet J, Addis A, Adcock I, Agache I, Agusti A, Alonso A, et al. Integrated care pathways for airway diseases (AIRWAYSICPS). Eur Respir J 2014;44:304-23.

[7] Blain H, Abecassis F, Adnet PA, Alomene B, Amouyal M, Bardy B, et al. Living Lab FallsMACVIA-LR: the falls prevention initiative of the European Innovation Partnership on Active and Healthy Ageing (EIP on AHA) in Languedoc Roussillon. Eur Geriatr Med 2014;5:416-25.

[8] La Charte Région Languedoc Roussillon Handicap - www.convergence-Ir.fr/evenement/1/ charte-region-Ir-handicap.pdf (consulté le 20 octobre 2014)

[9] Palazzo C, Ravaud JF, Trinquart L, Dalichampt M. Ravaud P. Poiraudeau S. Respective contribution of chronic conditions to disability in France: results from the National DisabilityHealth Survey. PLoS One 2012;7:e44994.

[10] Reynard F, Vuadens P, Deriaz O, Terrier P. could local dynamic stability serve as an early predictor of falls in patients with moderate neurological gait disorders? A reliability and comparison study in healthy individuals and in patients with paresis of the lower extremities. PLOS One 2014;9:e100550.

[11] Lebec 0, Ben Ghezala MW, Leynart V, Laffont I, Fattal C, Devillers L, et al. High level functions for the intuitive use of an assistive robot. IEEE Int Conf Rehabil Robot 2013:2013:6650374.

[12] ARS 2012 - http://www.drees.sante.gouv.fr/ languedoc-roussillon.6766.html (Consulté le 20 octobre 2014).

[13] http://www.sanitaire-social.com/annuaire/ associations-pour-handicapes-languedocroussillon/LANG/24/rc/1 (Consulté le 20 octobre 2014)

[14] Laffont I, Julia M, Tiffreau V, Yelnik A, Herisson C, Pelissier J. Aging and sequelae of poliomyelitis. Ann Phys Rehabil Med 2010:53:24-33.

[15] Ribaud A, Tavares I, Viollet E, Julia M, Hérisson C, Dupeyron A. Which physical activities and sports can be recommended to chronic low back pain patients after rehabilitation? Ann Phys Rehabil Med 2013:56:576-94.

[16] van Dokkum L, Hauret I, Mottet D, Froger J, Métrot J, Laffont I. The contribution of kinematics in the assessment of upper limb motor recovery early after stroke. Neurorehabil Neural Repair 2014;28:4-12.

[17] Metrot J, Froger J, Hauret I, Mottet D, van Dokkum L, Laffont I. Motor recovery of the ipsilesional upper limb in subacute stroke. Arch Phys Med Rehabil 2013;94:2283-90.

[18] Metrot J, Mottet D, Hauret I, van Dokkum L, Bonnin-Koang HY, Torre K, et al. Changes in bimanual coordination during the first 6 weeks after moderate hemiparetic stroke. Neurorehabil Neural Repair 2013;27:251-9.

[19] Torre K, Hammami N, Metrot J, van Dokkum L, Coroian F, Mottet D, et al. Somatosensoryrelated limitations for bimanual coordination after stroke. Neurorehabil Neural Repair 2013;27:507-15.

[20] Varoqui D, Froger J, Pélissier JY, Bardy BG. Effect of coordination biofeedback on (re) learning preferred postural patterns in poststroke patients. Motor Control 2011;15:187205.

[21] Dupeyron A, Demattei C, Kouyoumdjian P, Missenard 0, Micallef JP, Perrey S. Neuromuscular adaptations after a rehabilitation program in patients with chronic low back pain: case series (uncontrolled longitudinal study). BMC Musculoskelet Disord 2013:14: 277.

[22] Benoit CE, Dalla Bella S, Farrugia N, Obrig H, Mainka S, Kotz SA. Musically cued gaittraining improves both perceptual and motor timing in Parkinson's disease. Front Hum Neurosci 2014:8:494.

[23] Laffont I, Dumas C, Pozzi D, Ruquet M, Tissier AC, Lofaso F, et al. Home trials of a speech synthesizer in severe dysarthria: patterns of use, satisfaction and utility of word prediction. | Rehabil Med 2007:39:399-404.

[24] Laffont I, Guillon B, Fermanian C, Pouillot S, Even-Schneider A, Boyer $F$, et al. Evaluation of a stair-climbing power wheelchair in 25 people with tetraplegia. Arch Phys Med Rehabil 2008;89:1958-64.

[25] Laffont I, Biard N, Chalubert G, Delahoche L, Marhic B, Boyer FC, et al. Evaluation of a graphic interface to control a robotic grasping arm: a multicenter study. Arch Phys Med Rehabil 2009:90:1740-8.

[26] Guiraud D, Azevedo Coste C, Benoussaad M, Fattal C. Implanted functional electrical stimulation: case report of a paraplegic patient with complete $\mathrm{SCl}$ after 9 years. J Neuroeng Rehabil 2014;11:15.

[27] Azevedo Coste C, Jovic J, Pissard-Gibollet R, Froger J. Continuous gait cycle index estimation for electrical stimulation assisted foot drop correction. J Neuroeng Rehabil 2014;11:118

[28] van Dokkum L, Mottet D, Bonnin-Koang HY, Metrot J, Roby-Brami A, Hauret I, et al. People post-stroke perceive movement fluency in virtual reality. Exp Brain Res 2012;218:1-8.

[29] Perrey S. Possibilities for examining the neural control of gait in humans with fNIRS. Front Physiol 2014;5:204.

[30] Derosière G, Dalhoumi S, Perrey S, Dray G, Ward T. Towards a near infrared spectroscopy-based estimation of operator attentional state. PLoS One 2014;9:e92045

[31] Picard R. Pertinence et valeur du concept de Laboratoire vivant (Living Lab) en santé et économie [Pertinence and value of the Living Lab concept for health care and independent living]. Rapport CGIET, n² 2010/46/CGIET/SG. Paris: Conseil Général de l'Industrie, de l'Énergie et des Technologies; Ministry of Economy, Finances and Industry; 2011. 